\title{
Actividades de enfermería para la satisfacción de necesidades familiares en cuidado intensivo adulto: una revisión integrativa
}

Nursing care activities addressing family care needs in adult intensive care units: An integrative review

Atividades de enfermagem para atender às necessidades familiares em terapia intensiva para adultos: uma revisão integrativa

Como citar este artículo: Ardila Suárez Edinson Fabian, Arredondo Holguín Edith del Socorro. Actividades de enfermería para la satisfacción de necesidades familiares en cuidado intensivo adulto: una revisión integrativa. Revista Cuidarte. 2021; 12(1):e1229. http://dx.doi.org/10.15649/cuidarte.1229

Revista Cuidarte

doi) Rev Cuid. Ene. - Abril. 2021; 12(1): e1229 http://dx.doi.org/10.15649/cuidarte.1229

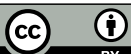

E-ISSN: 2346-3414

(1) Edinson Fabian Ardila Suárez ${ }^{1}$

(1) Edith del Socorro Arredondo Holguín ${ }^{2}$

1 Facultad de Enfermería, Universidad de Antioquia. Medellín, Colombia. E-mail: edinson.ardila@udea.edu.co

2 Facultad de Enfermería, Universidad de Antioquia. Medellín, Colombia. E-mail: edith.arredondo@udea.edu.co

\section{Resumen}

Introducción: la unidad de cuidado intensivo es un área hospitalaria donde se atienden personas con gravedad en su estado de salud, sin embargo la complejidad de la atención no está dada solamente por este aspecto sino por las necesidades de cuidado de la familia; la cual debe ser vista como una unidad; actualmente existe poca literatura sobre las intervenciones para el cuidado en esta área pues existe un trabajo interdisciplinario dentro de ella, siendo necesario un acercamiento al cuidado empírico que realiza enfermería en este entorno. Objetivo: identificar las actividades de cuidado de enfermería para la satisfacción de las necesidades de la familia en unidades de cuidado intensivo adulto. Metodología: se realizó una revisión integrativa fundamentada en los parámetros de Whittermore y Knafl, de artículos publicados entre 2008 y 2018, en las bases de datos: EBSCOhost, LILACS, MEDLINE, Science Direct, Scielo, Google Académico y Cochrane Library, los resultados se organizaron de acuerdo a la declaración PRISMA. Resultados: se incluyeron 16 artículos derivados de investigaciones cuantitativas, cualitativas y de revisiones; los cuales describen actividades para el cuidado de la familia de adultos. Las actividades encontradas se organizan a partir de la propuesta que Nancy Molter hace acerca de las necesidades de Seguridad, Información, proximidad, confort y apoyo, que la familia presenta en la unidad de cuidado intensivo. Conclusión: el cuidado de las necesidades de la familia de adultos en las unidades de cuidados intensivos permite abarcar al paciente y su familia como un ser integral y unitario, esto con el fin de mantener la salud familiar y prevenir crisis en las mismas.

Palabras clave: Unidades de Cuidados Intensivos; Salud de la Familia, Atención de Enfermería; Adulto.

Recibido: 28 de abril de 2020 Aceptado: 16 de septiembre de 2020 Publicado: 19 de febrero de 2021 $\square *$ Correspondencia

Edinson Fabian Ardila Suárez

E-mail: edinson.ardila@udea.edu.co 


\section{Nursing care activities addressing family care needs in adult intensive care units: An integrative review}

\section{Abstract}

Introduction: the intensive care unit is a hospital area where people with serious health conditions are cared for, however the complexity of care is not only given by this aspect but by the family's care needs; which must be seen as a unit; Currently, there is little literature on care interventions in this area because there is interdisciplinary work within it, requiring an approach to the empirical care that nursing performs in this setting. Objective: to identify nursing care activities to satisfy family needs in adult intensive care units. Methodology: an integrative review was carried out based on the Whittermore and Knafl parameters, of articles published between 2008 and 2018, in the databases: EBSCOhost, LILACS, MEDLINE, Science Direct, Scielo, Google Scholar and Cochrane Library, the results were organized according to the PRISMA declaration. Results: 16 articles derived from quantitative, qualitative and review research were included; which describe activities for the care of the adult family. The activities found are organized based on the proposal that Nancy Molter makes about the needs of Security, Information, proximity, comfort and support that the family presents in the intensive care unit. Conclusion: taking care of the needs of the adult family in intensive care units allows the patient and his family to be encompassed as an integral and unitary being, in order to maintain family health and prevent crises in them.

Keywords: Intensive Care Units; Family Health; Nursing Care; Adult.

\section{Atividades de enfermagem para atender às necessidades familiares em terapia intensiva para adultos: uma revisão integrativa}

\section{Resumo}

Introdução: a unidade de terapia intensiva é uma área hospitalar onde são atendidas pessoas com graves condições de saúde, porém a complexidade do atendimento não é dada apenas por esse aspecto, mas pelas necessidades de cuidado da família; que deve ser visto como uma unidade; Atualmente, há pouca literatura sobre intervenções assistenciais nessa área, pois existe um trabalho interdisciplinar, exigindo uma abordagem ao cuidado empírico que a enfermagem realiza nesse cenário. Objetivo: identificar as atividades de cuidados de enfermagem para satisfazer as necessidades familiares em unidades de terapia intensiva de adultos. Metodologia: foi realizada uma revisão integrativa com base nos parâmetros Whittermore e Knafl, a partir de artigos publicados entre 2008 e 2018, nas bases de dados: EBSCOhost, LILACS, MEDLINE, Science Direct, Scielo, Google Scholar e Cochrane Library; organizado de acordo com a declaração PRISMA. Resultados: 16 artigos derivados de pesquisas quantitativas, qualitativas e de revisão foram incluídos; que descrevem atividades para o cuidado da família adulta. As atividades encontradas são organizadas com base na proposta de Nancy Molter sobre as necessidades de segurança, informação, proximidade, conforto e apoio que a família apresenta na unidade de terapia intensiva. Conclusão: 0 atendimento às necessidades da família adulta em unidades de terapia intensiva permite que o paciente e sua família sejam englobados como um ser abrangente e unitário, a fim de manter a saúde da família e prevenir crises nelas.

Palabras clave: Unidades de Terapia Intensiva; Saúde da Família; Cuidados de Enfermagem; Adulto. 


\section{Introducción}

El padecimiento de la enfermedad crítica implica para los sistemas de salud la demanda de un mayor número de unidades de cuidados intensivos (UCI), recurso humano calificado e infraestructura de alta complejidad para poder brindar un cuidado de calidad e integral tanto a las personas hospitalizadas en dichas unidades como a su familia'; en la actualidad el cuidado de la persona críticamente enferma hospitalizada en la UCl es una prioridad en los sistemas de salud del mundo y de Colombia. Patologías cardiacas cerebrales, pulmonares, posquirúrgicas llevan a la personas a requerir este tipo de atención, un importante número de estas presentan necesidades humanas complejas y mueren, significando esta experiencia un gran nivel de sufrimiento y costos psicológicos, físicos, sociales y espirituales para el paciente, la familia y los profesionales ${ }^{2-4}$.

Needham ${ }^{5}$ concluye que la hospitalización en la UCl afecta no sólo al paciente, sino a su familia, la cual experimenta ansiedad, depresión, stress; ruptura en los roles que desempeña en el grupo y en las relaciones con otros miembros de la familia. Durante la hospitalización de un miembro de la familia en la $\mathrm{UCl}$ se dan alteraciones en el proyecto de vida del grupo y en su calidad de vida; experimentando fatiga, alteración del sueño y trastornos alimentarios; los cuales pueden durar el tiempo de la hospitalización o años después ${ }^{6}$; además sentimientos de ambivalencia, pérdida, amenaza de muerte y duelo, angustia, dolor, sufrimiento, frustración, preocupación, depresión, ansiedad, intranquilidad e impotencia, planteándose así un reto para los profesionales de enfermería que se desempeñan en la $\mathrm{UCl}$; pues el cuidado se convierte en un acto complejo que no está determinado únicamente por las condiciones críticas del paciente, sino por las necesidades de cuidado que experimentan las familias; además de las barreras propias; estudios en departamento Colombiano mostraron que las principales dificultades para brindar atención a la familia en la $\mathrm{UCl}$ está relacionada con el sistema, la estructura organizacional y falta de iniciativas o políticas en estos lugares?

La relación entre la enfermedad crítica del adulto y la alteración de la salud de la familia es clara, en la medida que se comprende ésta como un sistema con estrechos vínculos compuesto por subsistemas (cada uno de los miembros que integran la familia); a múltiples estresores que pueden llegar a afectar su estabilidad y bienestar. Frente a ello el profesional de enfermería debe considerar no sólo la respuesta individual al estresor que viven los subsistemas sino la respuesta del sistema; y cómo funcionan las líneas de defensa y la resistencia de la familia frente a la tensión colectiva con el fin de mantener su salud 9 ; esto a través de intervenciones de enfermería; en este sentido, Newman ${ }^{10}$ propone que las intervenciones de cuidado de enfermería tienen por objeto reducir los factores que generan tensión y las condiciones adversas, con el fin de mantener la salud, la adaptación y la estabilidad.

Las intervenciones de cuidado de enfermería tienen por objeto reducir los factores que generan tensión y las condiciones adversas, con el fin de mantener la salud, la adaptación y la estabilidad.

Desde la profesión de enfermería la investigación se ha orientado a la identificación de las necesidades de la familia propuestas por Molter ${ }^{11}$ y sus dimensiones validadas por Leske ${ }^{12}$ : seguridad, información, proximidad, confort y apoyo; sin embargo, existe muy poca producción científica sobre la realización de dichas intervenciones en el contexto latinoamericano. Ahora bien, partiendo del lenguaje estandarizado de intervenciones de enfermería NIC, estas están compuestas por actividades que orienten el logro de los objetivos propuestos para cada una; por lo tanto es necesario conocer y mostrar desde el cuidado empírico cuales es el cuidado que los profesionales de enfermería brindan a la familia en $\mathrm{UCl}$ adulto. En este orden de ideas, el 
objetivo de esta revisión fue identificar cuáles son las actividades de cuidado de enfermería que se realizan para la satisfacción de las necesidades familiares de adultos hospitalizados en UCI.

\section{Materiales y Métodos}

Se realizó una revisión integrativa de la literatura de alcance descriptivo basada en los parámetros de Whittermore y Knafl' ${ }^{13}$ durante 6 meses (abril - septiembre/2018), la cual consistió en 5 fases: identificación del problema y pregunta de investigación, búsqueda de la literatura, evaluación de los datos, análisis de los datos y presentación de los resultados.

Durante el periodo comprendido entre abril-septiembre de 2018 se realizó una búsqueda de literatura en las bases de datos: EBSCOhost, LILACS, MEDLINE, Science Direct, SciELO-Scientific Electronic Library Online, Google Académico y Cochrane Library; se utilizó la estrategia PICO para definir los criterios de búsqueda, en la cual se incluyeron los siguientes términos MeSH, DeCs en idiomas español, inglés y portugués junto a los operadores boleanos: "Familia" AND "Cuidado de enfermería" AND “Unidad de Cuidados Intensivos" AND NOT "neonato" AND NOT "pediátrico". Como límites de la búsqueda se tuvo en cuenta: artículos en idiomas español, inglés y portugués; publicaciones entre 2008 y 2018 y con resumen y texto completo disponible; como criterios de inclusión se tuvo en cuenta: Familias de adultos, Artículos publicados, estudios cuantitativos y cualitativos, proyectos de maestrías y tesis de doctorados en repositorios on-line. la evaluación de los datos se realizó bajo el análisis sistemático de los datos el cual permitió la interpretación en fuentes primarias, se contó con un segundo revisor de manera independiente, con el fin de evaluar la evidencia para luego organizarlas según las categorías encontradas; los artículos duplicados solamente se tuvieron en cuenta una sola vez. (Ver. Figura 1)

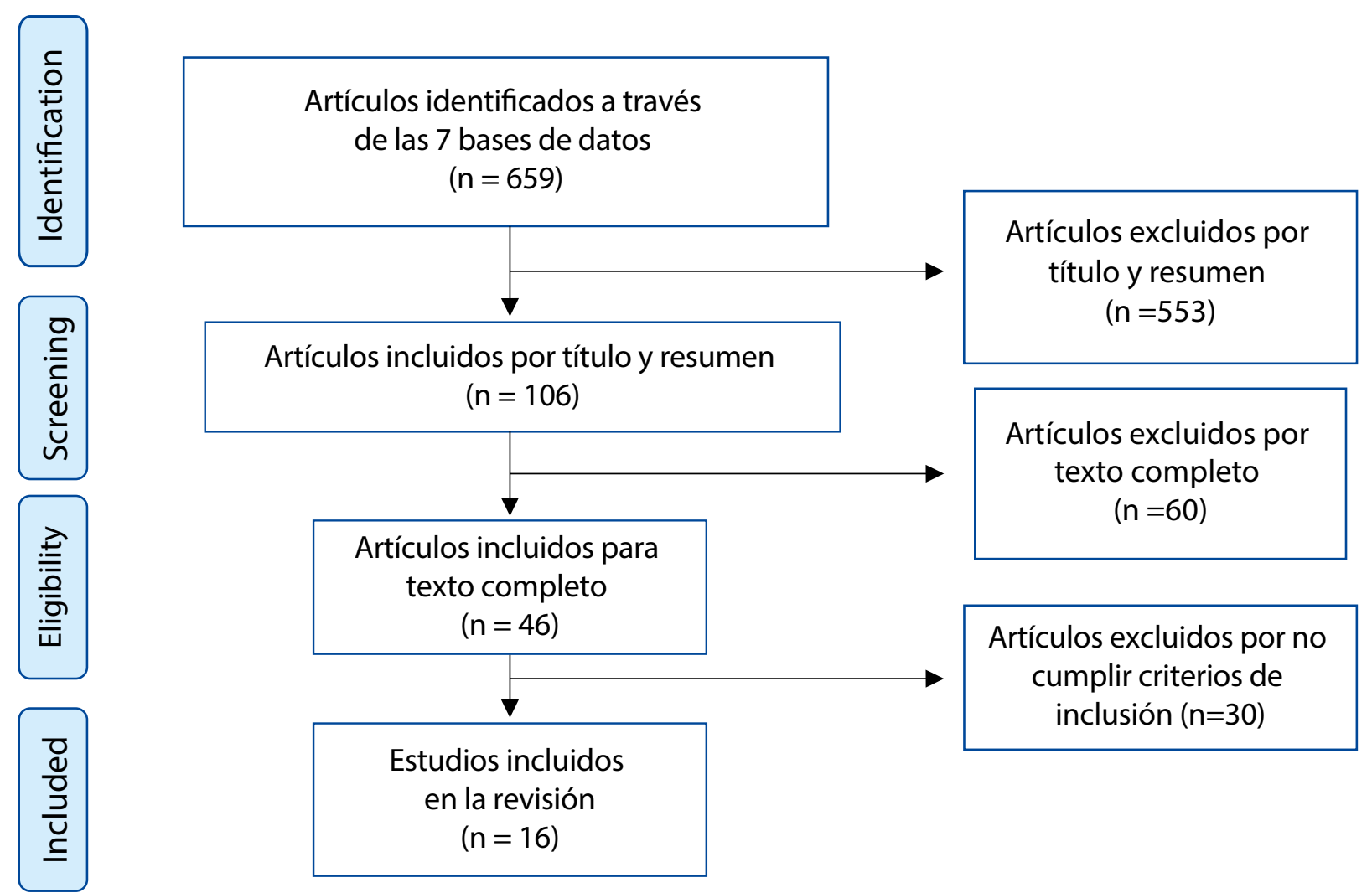

Figura 1. Flujograma para la selección e inclusión de los artículos

Fuente: Declaración PRISMA para publicación de revisiones sistemáticas y meta-análisis ${ }^{14}$ 


\section{Resultados}

Caracterización de los artículos: un total de 659 artículos fueron identificados, se evaluaron 106 por título y resumen, se incluyeron 46 para lectura de texto completo y se seleccionaron 16 para la revisión que incluían actividades de cuidado para la familia de adultos en UCl. Para la recolección, organización y análisis de los datos se diseñó un instrumento Ad Hoc, el cual contenía información sobre el artículo así: revista, autores, año, palabras clave, descriptores, idioma, lugar de investigación, periodo de análisis, año de publicación, objetivos, justificación, diseño, conclusiones y observaciones.

Los artículos fueron publicados en las siguientes revistas: Rev. Salud, Investigación en enfermería: Imagen y Desarrollo, Critical Care Nursing Quarterly, Journal of palliative medicine, Iranian Journal of Nursing and Midwifery Research, American Journal of Critical Care, Pensar Enfermagem, Australian Critical Care, JBI The Joanna Briggs institute, Art \& Science critical care, Heart and Lung, Enfermería Intensiva, Enfermería Universitaria, Journal of Research in Nursing, Revista Latinoamericana de enfermagem, Clinical Care Nursing Clinic.

En lo relacionado con los años de publicación, se encontró evidencia desde 2010 en adelante hasta 2017, exceptuando 2011, en la distribución de los idiomas se encuentra de predominancia Inglés 62.5\%, Español 25\% y Portugués 12,5\%; Los estudios fueron realizados en EEUU, Australia, Irán, Brasil, España, Inglaterra y Colombia con diversos diseños metodológicos de investigación (Tabla 1. Artículos incluidos para la revisión integrativa).

Tabla 1. Artículos seleccionados para la revisión integrativa entre 2010-2017

\begin{tabular}{llllll}
\hline & Autor & País & Año & \multicolumn{1}{c}{ Diseño Metodológico } & Ref. \\
\hline 1 & García et al. & España & 2010 & Investigación acción Participativa & 15 \\
2 & Davidson \& col. & EEUU & 2010 & Ensayo Clínico Controlado & 16 \\
3 & Yousefi \& col. & Irán & 2012 & Ensayo Clínico Controlado & 17 \\
4 & Achury \& col. & Colombia & 2013 & Investigación propuesta teórica & 6 \\
5 & Contreras \& col. & Colombia & 2014 & Revisión de literatura & 18 \\
6 & Aslakson et al. & EEUU & 2014 & Revisión sistemática & 19 \\
7 & Khalaila R. & Inglaterra & 2014 & Revisión de literatura & 20 \\
8 & Adams \& col. & EEUU & 2014 & Investigación cualitativa tipo & 21 \\
9 & Bernal \& col. & Colombia & 2014 & Investigación propuesta teórica & 22 \\
10 & Freitas KS \& col. & Brasil & 2015 & Estudio de validación documental & 23 \\
11 & Bandari et al. & Irán & 2015 & Estudio Descriptivo, transversal & 24 \\
12 & Ferreira \& col. & Brasil & 2015 & Revisión sistemática & 25 \\
13 & Torke et al. & EEUU & 2016 & Ensayo clínico controlado & 26 \\
14 & Mitchell \& col. & Australia & 2016 & Revisión integrativa & 27 \\
15 & Kynoch K \& col. & Australia & 2016 & Revisión sistemática & 28 \\
16 & Young L \& col. & EEUU & 2017 & Revisión basada en la evidencia & 29 \\
\hline
\end{tabular}

Fuente: Elaboración propia 


\section{Síntesis de los resultados}

Se realizó lectura en profundidad de los diferentes artículos seleccionados basados en el objetivo principal, además teniendo en cuenta la propuesta teórica de Nancy Molter sobre la investigación de las necesidades de la familia en la $\mathrm{UCl}$ y de acuerdo a lo emergido en la revisión, se organizaron las actividades de enfermería para el cuidado de la familia de acuerdo a las 5 necesidades principales que presentan dentro dicho entorno (seguridad, información, proximidad, confort y apoyo). (Ver Figura 2. Organización del cuidado a la familia en UCl adultos)

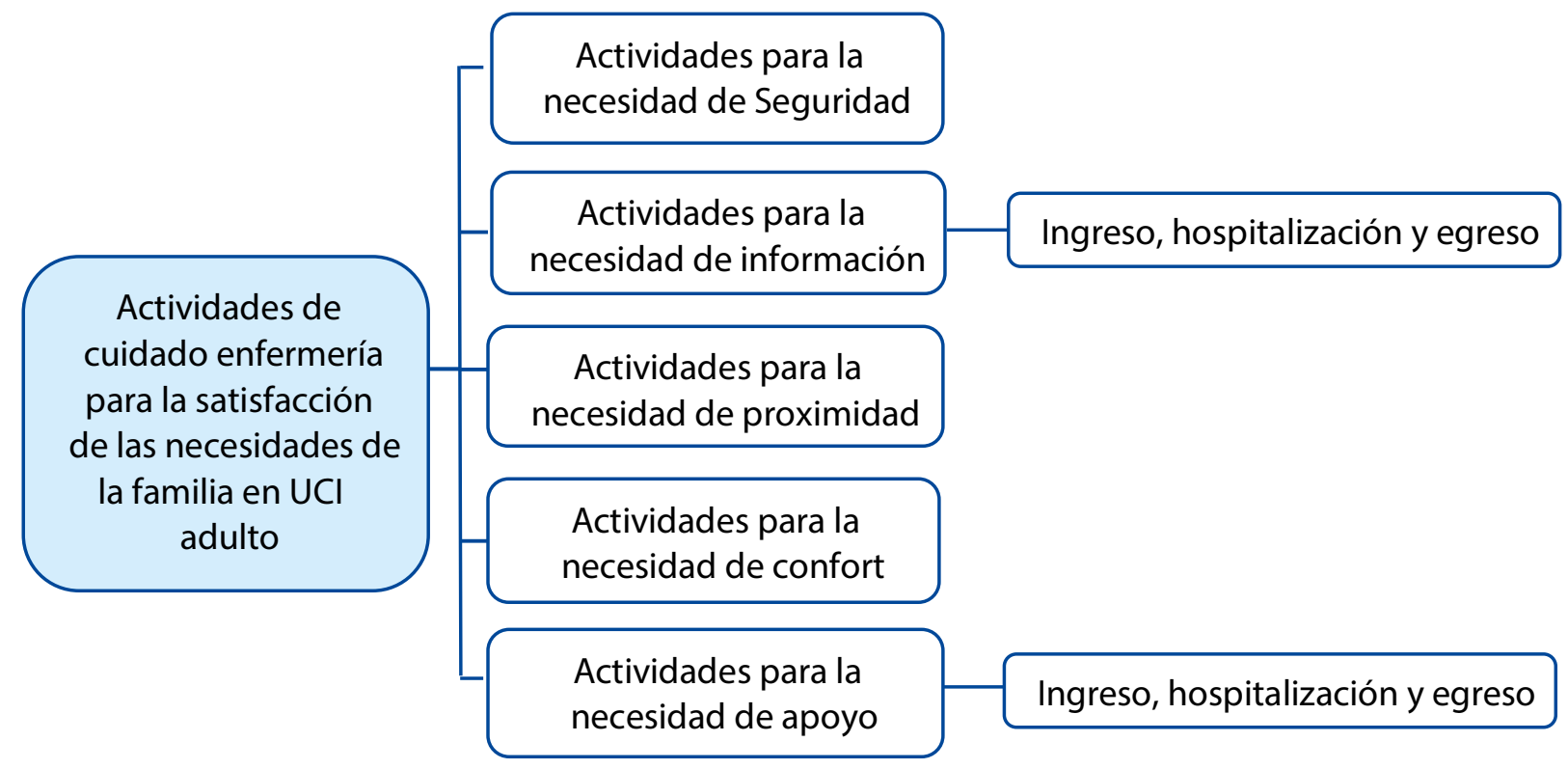

Figura 2. Organización del cuidado a la familia en $\mathrm{UCl}$ adultos

Fuente: Elaboración propia

Actividades de cuidado para brindar seguridad a la familia $6,16,17,20,21,23,25,27,28$ El interés por la seguridad de la familia en la UCl es cada vez mayor; los estudios reportan que la percepción de seguridad que experimenta la familia en la UCl, está dada por: la interacción humana que el personal de salud propicia y por la posibilidad que ésta ve de participar en el cuidado de la higiene, comodidad y la satisfacción de necesidades básicas a la cabecera de la cama de su ser querido; la participación de la familia en las rondas del personal de salud, en la observación de la reanimación cardiopulmonar y procedimientos invasivos, en la entrega de turno, que se le permita participar en la toma de decisiones sobre la no reanimación y pueda estar durante la evaluación

La familia se siente segura cuando logra percibir un orden general en el entorno de la unidad y observa como el personal de salud suple con todo profesionalismo las necesidades del paciente. de la muerte cerebral; también experimentan seguridad cuando encuentran un entorno amable y abierto que les permita su estancia en la UCI. La familia se siente segura cuando logra percibir un orden general en el entorno de la unidad y observa como el personal de salud suple con todo profesionalismo las necesidades del paciente. La seguridad para la familia se experimenta en la medida que el personal de salud muestra interés por el estado del paciente, y se acerca con franqueza mirando a los ojos, llamándolos por su nombre, respetando su confidencialidad y considerando las preferencias de ellos en el cuidado del paciente, esto le permite sentir a la familia que ellos les importan al equipo de salud. Todas estas actividades son posibles gracias a la apertura que los profesionales de la salud han tenido por la atención en salud a las familias en una UCl de puertas abiertas. 
Actividades de cuidado para brindar información a la familia ${ }^{15-25,28}$. Los profesionales de la salud en la UCI han propuesto diversidad de intervenciones dirigidas a la satisfacción de la necesidad de información de la familia. Proponen que deben darse desde el momento que ingresa el paciente a la UCl acerca de: la ubicación en los espacios físicos dentro de la UCl, el conocimiento de los equipos y sus alarmas, los medios existentes para comunicarse con el personal, tipo de procedimientos que se le realizaran al paciente y nombres de los miembros del equipo de salud de la $\mathrm{UCl}$.

La literatura reporta además que los profesionales de salud tienen estrategias como: entregar folletos con indicaciones y normas de comportamientos que debe tener la familia dentro de la $\mathrm{UCl}$, elaboración de tarjetas con los números telefónicos de la UCl y de las familia con el fin de comunicarse en el caso que la condición del paciente cambie, realizan sesiones educativas y conversatorios con los miembros de la familia, entregan directorios con el nombre del personal de salud de la $\mathrm{UCl}$, realizar conferencias acerca del ingreso, permanencia y alta del paciente, usan talleres de pintura y gráficos con el fin de educar a la familia en relación a las dinámicas de la UCl. Informar acerca del horario de la visita y educar a la cabecera del paciente a la familia frente al cuidado de necesidades básicas de su ser querido, es decir "aprender haciendo".

Es importante que el personal de salud siempre hable a la familia en términos que sean comprensibles, respondiendo honestamente las preguntas y aclarando las dudas e inquietudes sin dar falsas esperanzas ante el pronóstico de salud de su familiar, ni comparar con el estado de otros pacientes, evitando dar consejos no solicitados; también es importante entregar información sobre el estado del paciente al menos una vez al día, realizar sesiones de información; durante las visitas es importante que el personal clarifique información tanto al inicio como al final según la necesidad y en lo relacionado con el alta, traslado o egreso es necesario realizar conferencias de preparación para la misma.

Actividades de cuidado para brindar proximidad a la familia 6, 16-18, 20-23, 25, 27 . Para orientar el cuidado de enfermería hacia la satisfacción de la necesidad de proximidad la familia debe ser tenida en cuenta en los diferentes momentos durante la estancia hospitalaria manteniendo la presencia de los familiares cercanos al entorno del paciente, permitiendo la proximidad física y las manifestaciones de cariño la participación familiar en algunas acciones de cuidado básico, el uso de elementos de cuidado personal y estableciendo rutinas que afiancen la proximidad entre la familia y el paciente y explicando que el paciente puede escucharlos incluso estando en coma o bajo efectos de los anestésicos; otra manera consiste en mantener siempre un dialogo directo sobre la condición del paciente e implementar el uso del diario familiar como medio para registrar sus experiencias durante la hospitalización; también, es de vital importancia permitir la presencia de la familia en los últimos momentos de vida del paciente (despedida) y por último realizar contacto físico con los familiares cuando sea requerido.

Actividades de cuidado para brindar confort a la familia15,16,18,20,21,23,25. Para orientar el cuidado de enfermería hacia la satisfacción de la necesidad de confort, se debe tener en cuenta que dentro de los aspectos del confort físico se requieren cambios estructurales y/o de infraestructura sumado a modificaciones en las políticas institucionales; sin embargo desde el confort espiritual es muy importante la identificación y el respeto de la cultura del paciente y de los miembros de la familia, permitiéndoles que tengan privacidad sobre sus creencias y dejándoles disponer de objetos considerados sagrados dentro del entorno físico de la UCl; y en los casos requeridos permitir la utilización de tecnologías de la información para promover las conversaciones diarias. 
Actividades de cuidado para brindar apoyo a la familia 15,17,18,20,21,23-29 : Para orientar el cuidado de enfermería hacía la satisfacción de la necesidad de apoyo la familia debe ser tenida en cuenta desde el momento que ingresa el paciente a la $\mathrm{UCl}$ por lo tanto se recomienda realizar un encuentro con la familia durante las primeras 24 horas para identificar las principales necesidades, identificando los recursos cognitivos y sociales que les pueden dar soporte, crear grupos de apoyo entre los miembros de la familiar y el equipo interdisciplinar, realizar una reunión semanal con la familia para permitir la expresión de sentimientos, emociones y resolver dudas e inquietudes y sobre el estado del paciente; en lo relacionado con la visita es importante realizar excepciones en la medida que lo amerite; por otro lado, el apoyo puede ser visto desde aspectos como aceptación de las decisiones que hayan tomado los miembros de la familia en relación con la salud y futuro del paciente, estar con ellos en los momentos de crisis

El apoyo puede ser visto desde aspectos como aceptación de las decisiones que hayan tomado los miembros de la familia en relación con la salud y futuro del paciente. y en las reacciones inmediatas de preocupación, hablar sobre la posibilidad de la muerte del paciente y orientarlos sobre apoyo espiritual y la realización del mismo; la empatía juega un papel fundamental y por lo tanto es importante demostrarla tanto al paciente como a la familia de esta manera se lograr escuchar y oír con mucha atención a la familia siendo optimistas ante las situaciones presentadas y usando el sentido del humor cuando las condiciones lo permitan y en la medida de lo posible empoderar a los miembros de la familia en su autocuidado.

\section{Discusión}

Las actividades que los profesionales de enfermería realizan para el cuidado de la familia reportados en la literatura revisada en el presente estudio, se organizaron desde el criterio teórico propuesto por Molter $\mathrm{N}$ y Leske J; quienes identificaron que la familia en la UCI tiene necesidad de seguridad, de información, de apoyo, de confort y de proximidad.

Actividades para suplir la necesidad de seguridad: Según el Ministerio de la Protección Social, la seguridad del paciente se define como el conjunto de elementos estructurales, procesos, instrumentos y metodologías basadas en la evidencia científica, que buscan minimizar el riesgo de sufrir un evento adverso relacionado con la atención en salud o mitigar sus consecuencias. Dentro de las acciones institucionales por establecer la política de seguridad, se propone la implementación sistemática de herramientas prácticas, como instituir una cultura de seguridad justa, educativa no punitiva, reducir la incidencia de incidentes y eventos adversos y construir una alianza con el paciente y su familia; que logre incentivar en ellos preguntas acerca de su enfermedad, su cuidado y los riesgo de la atención y promover su participación en el cuidado y en la prevención de incidentes ${ }^{30}$.

Milos et al; plantean que dentro de la prescripción de cuidados de enfermería, se debe considerar que la persona tiene entre otras la necesidad de seguridad; vista como la capacidad de independencia, estabilidad, protección de peligros ambientales y la ausencia del miedo y la ansiedad frente a la atención de salud. La satisfacción de la necesidad de seguridad es aquella percepción que permite al paciente y a su familia sentirse apoyado, estable, independiente y protegido de los peligros ambientales; y le permite estar en la $\mathrm{UCl}$ sin miedo o ansiedad. Cuando desde un servicio de enfermería trabaja en la satisfacción de la necesidad de seguridad, ésta le ayuda a los pacientes y sus familias a atenuar sentimientos de desprotección y vulnerabilidad cuando se está hospitalizado; desde la cultura de seguridad no sólo hay que preocuparse por los 
daños o eventos adversos; sino por las preferencias, deseos, necesidades y expectativas de éstos frente a los tratamientos y procedimientos, con miras a aminorar la sensación de inseguridad que los acompaña. Es importante que desde la intervención de valoración del estado de la familia de los pacientes hospitalizados en UCI los profesionales de enfermería pregunten por las preferencias, los deseos, las necesidades espirituales y las expectativas de los pacientes y sus familias frente a los tratamientos y procedimientos ${ }^{31}$.

Sánchez et al; concluyen en su estudio, que la familia en la UCl determina como prioritaria la necesidad de seguridad; y entienden ésta como un estado de tranquilidad, que les proporciona percibir que el cuidado que recibe su paciente está bien; que el personal de salud facilita el acceso para que la familia este al lado de su ser querido y que además se disponga de instalaciones físicas para el descanso que requieren para afrontar las largas esperas. Concluyen que se percibe La familia en la UCI determina como prioritaria la necesidad de seguridad; $y$ entienden ésta como un estado de tranquilidad, que les proporciona percibir que el cuidado que recibe su paciente está bien. inseguridad cuando la atención en la UCI se centra en la fisiopatología del paciente y se corta todo tipo de comunicación e interacción con la familia ${ }^{32}$.

Actividades para suplir la necesidad de información: Anderson et al. Menciona que la información es un elemento que ayuda en la UCI para que haya una atención más integral y humana del paciente y su familia; en la medida que contribuye a aceptar el pronóstico de una enfermedad crítica, su evolución y/o desenlace en la posible muerte de un familiar o la preparación para el alta cuando hay una recuperación de la salud ${ }^{33}$. Sánchez et al; concluye que para la familia es muy relevante recibir información de parte del equipo de salud; pero requieren de ésta sea comprensible, veraz, sincera y adecuada en el tiempo; sin embargo encuentran que las familias perciben un déficit de formación por parte del personal de salud de las UCl; esto debido a falta de la competencia de comunicación e información que tienen, el desinterés, la falta de tiempo, la transposición de papeles y funciones dentro del equipo de salud. En relación con los profesionales de enfermería se encuentra que éstos desisten de la labor de informar a la familia, aludiendo que es una labor médica que no les compete; y a su vez en el equipo interdisciplinario en general se evidencia un desequilibrio en las relaciones de poder lo que conlleva a que se den cesiones, restricciones o déficit de información ${ }^{32}$.

Gaeeni $\mathrm{M}$ et al. Plantea que recibir información del proceso de admisión en la UCl, estancia y cuidado de la propia familia es fundamental para la evolución del paciente y la disminución del stress de la familia, por lo que consideran que deben darse de manera constante y gradual durante la hospitalización. Para ello se requiere que sea veraz, genuina, basada en el conocimiento científico, que tenga un lenguaje sencillo para que sea comprensiva para todos los integrantes de la familia. Dentro del equipo de salud el profesional de enfermería dispone de una mayor disposición y habilidad para comprender el estado emocional de la familia y ello hace que las personas tengan una mayor esperanza y confianza durante el proceso de hospitalización en la UCl. También concluyen en su estudio que intervenir desde la información y la comunicación es la manera más óptima de afrontar la vivencia estresante de la UCl con una enfermedad crítica. Que las familias buscan la verdad y la honestidad en el equipo de salud y encontrarlas reduce la ansiedad y los altos niveles de stress $^{34}$.

En los actuales tiempos la tecnología ha sido de gran apoyo para realizar intervenciones en relación a la información que se brinda a la familia. La elaboración de páginas web y blogs en las 
UCI permiten organizar y sistematizar la información que se brinda a la familia y a su vez lograr que en la medida que ésta la reciba realice devoluciones y recomendaciones a ésta a partir de sus necesidades y percepciones. En la web se recogen preguntas de la familia, se organizan contenidos en relación al funcionamiento de la $\mathrm{UCl}$, la atención del paciente y el apoyo social de la familia, consejos de autocuidado ${ }^{35}$.

Actividades para suplir la necesidad de proximidad: De acuerdo con De Beer ${ }^{36}$ la proximidad se refiere a ser flexible con respecto al acceso de la familia al entorno del paciente y hacer que sea más fácil (tanto física como psicológicamente) el estar cerca de su ser querido; dicha cercanía le permite a los miembros de la familia monitorear la situación, rastrear la atención brindada y desarrollar confianza con el personal que brinda atención; esto también es acorde con lo encontrado por Shorofi et al. ${ }^{37}$ donde se encontró que la proximidad es una de las principales necesidades de la familia en la UCl adulto.

Abdel-Aziz et al. ${ }^{38}$ menciona que dentro de las principales dificultades que tiene el personal de la UCI para disminuir el estrés de las familias consiste en la poca satisfacción las necesidades de proximidad; también encontró que los profesionales de enfermería consideran importante para las familias aspectos como: hablar sobre los planes de traslado o alta, ver el paciente frecuentemente y tener puntualidad en los horarios

Las principales dificultades que tiene el personal de la UCI para disminuir el estrés de las familias consiste en la poca satisfacción las necesidades de proximidad. de visitas; todos estos aspectos están directamente relacionados con las actividades encontradas en este estudio, las cuales tienen como finalidad tener en cuenta a la familia en los diferentes momentos de la estancia hospitalaria del paciente, integrándola en las dinámicas y favoreciendo la confianza sobre el cuidado brindado.

Actividades para suplir la necesidad de confort: Kolkaba ${ }^{39}$ define el confort como un concepto holístico, subjetivo y multidimensional, influenciado por los contextos físicos, ambiental, social y psicoespiritual, que cambia con el tiempo y con el espacio; es el resultado de las interacción de los individuos consigo mismo, las personas que los rodean y las situaciones que enfrentan en la enfermedad y el proceso de atención en salud; en éste sentido brindar confort a la familia produce alivio físico-espiritual, reduce la aflicción, la angustia inmediata y mejora el bienestar percibido durante la hospitalización en la UCl.
Brindar confort a la familia produce alivio físico-espiritual, reduce la aflicción, la angustia inmediata y mejora el bienestar percibido durante la hospitalización en la UCI.

La investigación entre la satisfacción de las necesidades de la familia y el confort es un tema poco explorado, encontrándose pocos estudios en la literatura; Shorofi et al. 2016 ${ }^{37}$ \& Liew et al. $2018^{40}$ demuestran que el confort de familia no es una prioridad para el profesional de enfermería en la UCl; Meneguin et al. ${ }^{41}$ tienen la hipótesis de que la religiosidad/ espiritualidad y las variables sociodemográficas de los miembros de la familia, así como el perfil de los pacientes, influyen en el confort y las necesidades percibidas de los mismos.

Actividades para suplir la necesidad de apoyo: De acuerdo con Bautista et al.$^{42}$ el apoyo se define como el soporte recibido por el familiar en la interacción de cuidado con el personal de enfermería, a través de un trato cordial y amistoso, con calor humano, que da comodidad y confianza, muestra preocupación e interés por su sentir, tranquiliza la ansiedad y maneja la crisis situacional; también resalta que los familiares dentro de las instituciones hospitalarias valoran más los aspectos del cuidado, que tienen relación con ver al ser humano como holístico, 
donde comportamientos como la empatía, el afecto, el tacto como acción de apoyo y cuidado por parte del personal profesional de enfermería a cargo de su salud, son percibidos como un cuidado de calidad.

Wetzig ${ }^{43}$ en su estudio encontró que las familias de pacientes en UCl identificaron una necesidad de apoyo que abarcaba una gran de áreas, incluyendo orientación emocional, práctica espiritual y generación de relaciones en el entorno de la UCl; también el apoyo externo fue identificado como importante; aunque encuentran que el personal de salud fue el principal apoyo durante el periodo de hospitalización; en este sentido el apoyo se acepta como multifacético e incluye el cuidado de los requisitos emocionales, físicos, culturales y espirituales de la familia.

\section{Conclusiones}

La literatura analizada en el presente estudio; plantea un panorama a los equipos de salud y en particular a la enfermería, frente al cuidado de la familia en la UCl; proponiendo actividades orientadas a la satisfacción de las necesidades de seguridad, información, proximidad confort y apoyo; como un aporte importante para contribuir a salud de la familia y el paciente como una unidad.

La seguridad y la información como necesidades importantes de la familia, permiten ser suplidas desde los profesionales de la salud y de enfermería a partir de la interacción humana y la comunicación; logrando que la familia durante el proceso de hospitalización participe en el cuidado de actividades básicas de su ser querido, aumente su autor reconocimiento, sienta confianza con el profesionalismo que cuidan a su familiar y elaboren el duelo frente a la muerte y baje niveles de estrés.

Los profesionales de enfermería deben comprender que la familia de la persona hospitalizada en $\mathrm{UCl}$ es un sujeto de cuidado con múltiples necesidades, y su satisfacción aporta a su salud y el bienestar de todos sus miembros; lo cual favorece la salud de la familia durante el proceso de hospitalización.

Conflictos de interés: los autores declaran no tener ningún tipo de conflicto de interés

Financiamiento: El presente trabajo se encuentra asociado a la investigación titulada: Percepción sobre actividades de enfermería para el cuidado de la familia en unidades de cuidados intensivos adultos en el departamento de Santander; realizada en el marco de la Maestría en Enfermería, Facultad de enfermería, Universidad de Antioquia; la investigación no cuenta con recursos para su realización, sin embargo el investigador Edinson Fabian Ardila Suárez cuenta con financiación para formación posgradual por parte del Departamento de Ciencia, Tecnología e Innovación COLCIENCIAS y la Gobernación de Santander (Convocatoria 771/ 2016) 


\section{Referencias}

1. Florez Torres IE, Montalvo Prieto A, Romero Massa E. Incertidumbre en cuidadores familiares de pacientes hospitalizados en unidades de cuidado intensivo. Investig Enferm Imagen Desarr. 2018;20(1):1-12. https://doi.org/10.11144/Javeriana.ie20-1.icfp

2. Van Mol M, Boeter GW, Verharen L, Kompanje EJO, Bakker J, Nijkamp MD. Patient- and family-centred care in the intensive care unit: a challenge in the daily practice of healthcare professionals. J Clin Nurs. 2017;26 (19-20):3212-23. https://doi:10.1111/jocn.13669

3. Girbau M.B, Monedero P, Centeno C. Good care for patients who die in intensive care units in Spain. A study based on international care quality indicators. Anales Sis San Navarra. 2017; 40 (3): 339-349. http://dx.doi.org/10.23938/assn.0026

4. Padilla-Fortunatti C, Rojas-Silva N, Amthauer-Rojas M, Molina-Muñoz Y. Necesidades de los familiares de pacientes críticos en un hospital académico de Chile. Enferm Intensiva. 2018;29 (1):32-40. https://doi:10.1016/j.enfi.2017.09.001

5. Needham DM, Davidson J, Cohen H, Hopkins RO, Weinert C, Wunsch H, et al. Improving long-term outcomes after discharge from intensive care unit: Report from a stakeholders' conference. Crit Care Med. 2012;40(2):502-9. https://doi.org/10.1097/CCM.0b013e318232da75

6. Achury S DM, Achury B LF. Participación familiar en el cuidado de pacientes críticos: una propuesta de fundamentación teórica. Investig Enferm Imagen Desarr. 2013;15(2):137-51. http://www.redalyc.org/articulo.oa?id=145229803008

7. Marquez Herrera M, Carrillo González GM. La experiencia del familiar de la Unidad de Cuidados Intensivos en Bucaramanga (Colombia): Un estudio fenomenológico. Rev Arch Med. 2015;15(1):95-106. https://doi.org/10.30554/archmed.15.1.720.2015

8. Neuman B, Fawcett J. The Neuman Systems Model. Fifth edit. Pearson editor. lowa; 2011. 37-70 p.

9. Harmon Hanson SM, Rowe Kaakinen J, Friedman MM. Enfoques teóricos para la enfermería familiar [CD-ROM] Familia y cuidado de enfermería. Bogotá: Universidad Nacional de Colombia; 1992.

10. Lawson TG. Modelo de sistemas. In: Raile Alligood M, editor. Modelos y teorias en enfermería. Octava edi. España: Elsevier España, S.L.; 2015. p. 271-92.

11. Molter NC. Needs of relatives of critically ill patients : A descriptive study. Hear Lung J Acute Crit Care. 1979;8(2):332-9. https://www.ncbi.nlm.nih.gov/pubmed/253712

12. Leske JS. Internal psychometric properties of the Critical Care Family Needs Inventory. Hear Lung J Acute Crit Care. 1991;20(3):236-44. https://www.ncbi.nlm.nih.gov/pubmed/2032860

13. Whittemore $\mathbf{R}$, Knafl K. The integrative review: updated methodlogy. J Adv Nurs. 2005;5(52):546-553. https://doi.org/10.1111/j.1365-2648.2005.03621.x

14. Moher D, Liberati A, Tetzlaff J, Altman DG, The PRISMA Group (2009) Preferred Reporting Items for Systematic Reviews and Meta-Analyses: The PRISMA Statement. PLoS Med 6(7): e1000097. https://doi.org/10.1371/journal.pmed.1000097

15. García Mozo A, Sánchez Roldán F, Amorós Cerdá SM, Balaguer Albarracín F, Díez Alcalde M, Durán Torres MT, et al. Desarrollo de una guía de atención a los familiares del paciente crítico. Enferm Intensiva. 2010;21(1):20-7. https://doi.org/10.1016/j.enfi.2009.07.001

16. Davidson J, Daly B, Brady N, Higgins P. Facilitated Sensemaking A Feasibility Study for the Provision of a Family Support program in the Intensive Care Unit. Crit Care Nurse Q. 2010;33(2):177-89. https://doi.org/10.1097/CNQ.0b013e3181d91369

17. Yousefi H, Karami A, Moeini M, Ganji H. Effectiveness of nursing interventions based on family needs on family satisfaction in the neurosurgery intensive care unit. Iran J Nurs Midwifery Res. 2012;17(4):296-300.

https://www.ncbi.nlm.nih.gov/pmc/articles/PMC3702150/ 
18. Contreras Moreno A, Palacios Espinosa X. Contribuciones de la Psicología al manejo interdisciplinario del paciente en Unidad de Cuidados Intensivos (UCI). Rev Univ Ind Santander Salud. 2014;46(1):47-60.

19. Aslakson R, Cheng J, Vollenweider D, Galusca D, Smith T, Pronovost P. Evidence-Based Palliative Care in the Intensive Care Unit: A Systematic Review of interventions. J Palliat Med. 2014;17(2):219-35. https://doi.org/10.1089/jpm.2013.0409

20. Khalaila R. Meeting the needs of patients' families in intensive care units. Art Sci Crit Care. 2014;28:37-44. https://doi.org/10.7748/ns.28.43.37.e8333

21. Adams JA, Anderson RA, Docherty SL, Tulsky JA, Steinhauser KE, Bailey DE. Nursing strategies to support family members of ICU patients at high risk of dying. Hear Lung J Acute Crit Care. 2014;43(5):406-15. https://doi.org/10.1016/j.hrtlng.2014.02.001

22. Bernal-Ruiz D, Horta-Buitrago S. Cuidado de enfermería para la familia del paciente crítico desde la teoría de la comprensión facilitada. Enfermería Univ. 2014;11(4):154-63. https://doi.org/10.1016/s1665-7063(14)70928-5

23. Freitas KS, Menezes IG, Mussi FC. Validación de la escala de confort para familiares de personas en estado crítico de salud. Rev Latinoam Enferm. 2015;23(4):660-8. https://doi.org/10.1590/0104-1169.0180.2601

24. Bandari R, Heravi-Karimooi M, Rejeh N, Mirmohammadkhani M, Vaismoradi M, Snelgrove S. Information and support needs of adult family members of patients in intensive care units : an Iranian perspective. J Res Nurs. 2015;20(5):401-22. https://doi.org/10.1177/1744987115591868

25. Ferreira Rodrigues Galinha de Sá F, Rebelo Botelho MA, Pererira Henriques MA. Cuidar da Família da Pessoa em Situação Crítica: A Experiência do Enfermeiro. Pensar Enferm. 2015;19(1):31-46.

26. Torke AM, Wocial LD, Johns S, Sachs G, Callaham Ch, Bosslet G, Et al. The Family Navigator: A Pilot intervention to Support Intensive Care Unit Family Surrogates. Am J Crit Care, 2016; 25:498-507. https://doi.org/10.4037/ajcc2016730

27. Mitchell M, Coyer F, Kean S, Stone R, Murfiel J, Dwan T. Patient, family- centred care interventions within the adult ICU setting: An integrative review. Aust Crit Care. 2016;29:17993. https://doi.org/10.1016/j.aucc.2016.08.002

28. Kynoch K, Chang A, Coyer F, McArdel A. The effectiveness of interventions to meet family needs of critically ill patients in an adult intensive care unit : a systematic review update. Joanna Briggs Inst. 2016;179-232. https://doi.org/10.11124/JBISRIR-2016-2477

29. Young LK, Mansfield B, Mandoza J. Nursing Care of Adult Hematopoietic Stem Cell Transplant Patients and Families in the Intensive Care Unit: An evidence-based Review. Crit Care Nurs Clin N Am. 2017;29:341-52. https://doi.org/10.1016/j.cnc.2017.04.009

30. Ministerio de la protección social. Lineamientos para la implementación de la política de seguridad del paciente. Colombia. 2008. Pág. 13 - 27.

31. Milos $\mathbf{P}$, Larraín Al. La vinculación ético-jurídica entre la gestión del cuidado y la gestión del riesgo en el contexto de la seguridad del paciente. Aquichan. 2015; 15(1): 141-153. http://dx.doi.org/10.5294/aqui.2015.15.1.13

32. Sánchez-Vallejo A, Fernández D, Pérez-Gutierrez A, Fernández-Fernández $M$. Análisis de las necesidades de la familia del paciente crítico y la opinión de los profesionales de la Unidad de Cuidados Intensivos. Rev. Med Intensiva. 2016; 40(9):527-540. https://doi.org/10.1016/j.medin.2016.03.005

33. Anderson W, Cimino JW, Ernecoff NC, Ungar A, Shotsberger K, Pollice L, et al. A multicenter study of key stakeholders' perspectives on communicating with surrogates about prognosis in intensive care units. Ann Am Thorac Soc. 2015; 12 (2): 142-152. 
34. Gaeeni M, Farahani M, Seyedfatemi N, Mohammadi N. Informational support to family member of intensive care unit patients: the perspectives of families an nurses. Global Journal of Health Science. 2015; 7(2): 8-16. https://doi.org/10.5539/gjhs.v7n2p8

35. Suen A, Butler R, Arnold R, Myers B, Witteman H, Cox Ch, et al. Developing the family support tool: an interactive, web-based tool to help families navigate the complexities of surrogate decisión making in ICUs. J Crit care. 2020; 56: 132-139. https://doi.org/10.1016/j.jcrc.2019.12.002

36. De Beer J, Brysiewicz P. Developing a theory of family care during critical illness. S Afr J Crit Care. 2019;35(1):19-24. https://doi.org/10.7196/SAJCC.2019.v35i1.388

37. Shorofi SA, Jannati Y, Moghaddam HR, Yazdani-charati J. Psychosocial needs of families of intensive care patients : Perceptions of nurses and families. Niger Med J. 2016; 57 (1):10-8. https://dx.doi.org/10.4103\%2F0300-1652.180557

38. Abdel-aziz AL, Sayed Ahmed ES, El-hakeem Younis GA. Family Needs of Critically III Patients Admitted to the Intensive Care Unit, Comparison of Nurses and Family Perception. Am J Nurs Sci. 2017;6(4):333-46. https://doi.org/10.11648/j.ajns.20170604.18

39. Kolcaba K, Tilton C, Drouin C. Comfort Theory A Unifying Framework to Enhance the Practice Environment. J Nurs Adm. 2006;36(11):538-44 https://doi.org/10.1097/00005110-200611000-00010

40. Liew S, Dharmalingan T, Muniandy R, Johnny N, Lily N. Need Domains of Family Members of Critically-ill Patients : A Borneo Perspective. Borneo J Med Sci. 2018;12(2):27-33

41. Meneguin S, de souza Matos TD, Miot HA, Pollo CF. Association between comfort and needs of ICU patients' family members: a cross sectional study. J Clin Nurs. 2019; 28(34):538-44. https://doi.org/10.1111/jocn.14644

42. Bautista Rodríguez LM, Arias Velandia MF, Carreño Leiva ZO. Percepción de los familiares de pacientes críticos hospitalizados respecto a la comunicación y apoyo emocional. Rev Cuid. 2016; 7(2):1297. http://dx.doi.org/10.15649/cuidarte.v7i2.330

43. Wetzig K, Mitchell M. The needs of families of ICU trauma patients: an integrative review. Intensive Crit Care Nurs [Internet]. 2017;41:63-70.

http://dx.doi.org/10.1016/j.iccn.2017.02.006. 